Encontro Nacional de

Economia Industrial e Inovação
Indústria e Desenvolvimento Econômico:

desafios e perspectivas

18 a 20 de setembro de 2018

Uberlândia - Minas Gerais

Área ABEIN: 5.7 - Inovações, Competências e Competitividade

\title{
Inovações tecnológicas e organizacionais: um ensaio teórico e uma análise empírica dos serviços de tecnologia da informação de Minas Gerais a partir da PINTEC ${ }^{12}$
}

Márcia Siqueira Rapini (CEDEPLAR/UFMG)

Vanessa Parreiras de Oliveira (IE/UNICAMP)

André Luiz da Silva Teixeira (UNIFAL e Cedeplar /UFMG)

Thiago Caliari (ITA)

\section{Resumo}

O presente estudo analisa, teórica e empiricamente, a interdependência entre inovação tecnológica (IT) e organizacional (IO) no setor de Tecnologia da Informação (TI). Para isso, são utilizados os dados da PINTEC para os períodos de 2009-2011 e 2012-2014, comparando Minas Gerais e Brasil. Os principais resultados empíricos são: (1) a combinação de IT com IO pelas empresas mineiras de TI reduz a percepção da rigidez organizacional como obstáculo; e (2) para as firmas que não realizaram IT, a IO é mais presente nas firmas mineiras do que nas brasileiras, na média.

Palavras-chave: inovação tecnológica, inovação organizacional, tecnologia de informação, Minas Gerais, PINTEC.

\begin{abstract}
The present study analyzes, theoretically and empirically, the interdependence between technological (IT) and organizational innovation (OI) in the Information Technology (IT) sector. For this, the data from Brazilian Innovation Survey from 2009-2011 and 2012-2014, comparing Minas Gerais and Brazil are used. The empirical results are: (1) the combination of IT with OI in firms reduces the perception of organizational rigidity as an obstacle; and (2) for firms that did not perform IT, the OI is most present in firms from Minas Gerais than from for Brazilian firms, on average.
\end{abstract}

Key-words: technological innovation, organizational innovation, information technology, Minas Gerais, Innovation Survey.

JEL Code: O32; L86

\footnotetext{
${ }^{1}$ Este artigo foi desenvolvido no âmbito do Projeto "Inovação organizacional e tecnológica no setor de tecnologia de informação - descrição, caracterização e análise em empresas de minas gerais”, financiado pela FAPEMIG (Processo APQ-03078-15).

2 Agradecemos à Edmara de Oliveira pelo auxilio na elaboração das tabelas.
} 


\section{1 - Introdução}

A importância do setor de serviços, seja no produto ou na geração de empregos, vem crescendo de forma expressiva desde metade do século passado. Em 2016 ele contribuía com 73,4\% do valor agregado nos países da União Europeia (Eurostat) e responde por quase $75 \%$ do PIB no Brasil, em 2017, de acordo com o IBGE.

Apesar de envolver um conjunto amplo e heterogêneo de sub-setores e segmentos, uma parte dos serviços é altamente inovativa, caracterizando-se pela intensa geração de informação e de novos conhecimentos. Os serviços de informação e comunicação caracterizam-se por um forte caráter de localização, decorrente da natureza tácita de parte considerável do conhecimento envolvido no processo de produção. Assim como na indústria, a tecnologia da informação vem contribuindo para a geração de valor dos serviços, acelerando o processo de inovação e de geração de novos conhecimentos e informações, bem como a criação de novas empresas e nichos de mercado. $\mathrm{O}$ conhecimento e a aplicação de tecnologias de informação é de fundamental relevância para o desenvolvimento de inovações tecnológicas no setor de serviços (KUBOTA, 2007). A literatura salienta também que os serviços de informação e comunicação contribuem para o incremento da produtividade das economias. Estudos como o de Mendonça et al. (2009) identificaram que a adoção de tecnologia da informação afeta positivamente a produtividade da mão-de-obra da indústria de transformação brasileira. Estes aspectos, por si só, justificam investigar mais à fundo a inovação das empresas deste setor.

O aumento de importância dos serviços vem exigindo novas formas de conceituação e de mensuração do seu valor e da avaliação do seu impacto na geração de valor nas atividades de outros setores (KON, 2004). Um exemplo deste esforço é exatamente a inclusão do setor de serviços na Pesquisa de Inovação (PINTEC), realizada pelo Instituto Brasileiro de Geografia e Estatística (IBGE), a partir de 2005. A PINTEC passa, portanto, a fornecer importantes evidências estatísticas sobre a inovação tecnológica em serviços. A partir de 2008, a Pesquisa passou também a mensurar uma parte da inovação organizacional, similar ao esforço realizado no âmbito de Pesquisa realizada na Europa, a Community Innovation Survey - CIS. Até então, a pesquisa perguntava apenas sobre a realização de "mudanças organizacionais".

Diversos estudos vêm indicando que as estratégias de inovação das empresas são melhor compreendidas considerando em conjunto as inovações tecnológicas e organizacionais. Para o setor de serviços estas últimas aparecem como muito relevantes, acontecendo, em muitos casos, isoladamente, ou seja, não vinculada às inovações tecnológicas (EVANGELISTA e VEZZANI, 2010). As inovações organizacionais, portanto, podem acontecer em conjunto, antes ou depois de inovações tecnológicas, sendo fundamentais para sustentar o desempenho das empresas e seu crescimento (NELSON e SAMPAT 2001).

Frente a este quadro de referência, são dois os objetivos do presente artigo. O primeiro objetivo é avançar teoricamente, propondo uma articulação entre inovação tecnológica e organizacional na literatura Econômica. O segundo objetivo é analisar empiricamente, para o setor de Tecnologia da Informação (TI), ) a relação entre inovação tecnológica e organizacional a partir da PINTEC para os períodos de 2009-2011 e 2012-2014.

Ademais desta introdução, o artigo possui mais 4 seções. A segunda seção é um esforço de avançar teoricamente na articulação entre inovação tecnológica e organizacional dentro da literatura econômica, fazendo uma releitura em duas dimensões. Uma primeira dimensão meso-institucional a partir do conceito de paradigmas tecno-econômicos e a segunda dimensão no âmbito da firma. A terceira seção discorre de forma breve sobre o setor de Tecnologias da Informação (TI) de Minas Gerais. A quarta seção realiza a a análise empírica, apresentando. primeiramente, a PINTEC realizada pelo IBGE e, em seguida, as análises dos dados desta para os triênios 2009-2011 e 2012-2014 para o setor de TI comparando Minas Gerais com o Brasil. A sexta seção conclui o trabalho. 


\section{Inovações Tecnológicas e Organizacionais: uma via de mão-de-dupla}

Essa seção se propõe a discutir a relação de interdependência entre as inovações tecnológicas e organizacionais, seguindo as definições da $3^{a}$ edição do Manual de Oslo ${ }^{3}$.

Segundo esse Manual, uma inovação tecnológica (IT) pode ser dividida em dois tipos: de produto e de processo. A Inovação de Produto é tida como "a introdução de um bem ou serviço novo ou significativamente melhorado no que concerne a suas características ou usos previstos. Incluemse melhoramentos significativos em especificações técnicas, componentes e materiais, softwares incorporados, facilidade de uso ou outras características funcionais" (OCDE/FINEP, 2005, p. 57). Já as inovações de processo remetem à "implementação de um método de produção ou distribuição novo ou significativamente melhorado. Incluem-se mudanças significativas em técnicas, equipamentos e/ou softwares" (OCDE/FINEP, 2005, p. 58).

Já uma inovação organizacional (IO) é definida como a implementação de um novo método organizacional nas práticas de negócios da empresa, na organização do seu local de trabalho ou em suas relações externas (OCDE/FINEP, 2005, p. 61). As inovações nas práticas de negócios referemse a novos procedimentos na condução do trabalho, como a adoção de nova formas de compartilhar e codificar conhecimentos internos e novas firmas de gerenciar cadeias de produção e fornecedores. Já as inovações na organização do local de trabalho referem-se a novas práticas quanto à distribuição de responsabilidades e poder de decisão entre os trabalhadores e também quanto à integração entre as atividades da empresa. Por fim, as inovações nas relações externas referem-se a novos meios para organizar relações com agentes externos, incluindo novos tipos de colaborações, subcontratações e integrações em cadeias.

Essas inovações organizacionais (IOs) se diferenciam de outras mudanças organizacionais pois as novas práticas organizacionais implementadas não devem ter sido utilizadas antes pela empresa e devem ser resultantes de uma decisão estratégica tomada pela gerência. Ademais, as IOs se assemelham às inovações tecnológicos de processo quanto aos objetivos (reduzir custos e melhorar a eficiência), mas se diferenciam especialmente quanto à atividade: as inovações de processo abarcam a implementação de novos equipamentos, softwares e procedimentos enquanto as IOs focam nas pessoas e na organização do trabalho (OCDE/FINEP, 2005, p. 66).

Todavia, o próprio Manual de Oslo já sugere uma primeira relação entre IT e IO. Segundo este, a implementação de um novo método de produção - i.e. um tipo de IT - pode, por um lado, induzir e demandar a realização de novas formas de trabalho em grupo - i.e. uma IO - ou, por outro lado, ser resultado de um novo método organizacional, como, por exemplo, a implementação de um sistema de controle de qualidade que leve a melhorias substanciais nos processos produtivos (OCDE/FINEP, 2005, p. 66). Portanto, a partir da própria definição das ITs e IOs já se tem uma relação dupla entre estas, a qual será melhor explorada, a seguir, em duas dimensões: (1) a partir da ideia de paradigma técnico-econômico; (2) da firma.

\subsection{IO versus IT no âmbito dos paradigmas técnico-econômicos}

Quanto à primeira dimensão, o conceito de paradigma técnico-econômico (PTE) refere-se à combinação de diferentes tipos de inovações inter-relacionadas - e.g. inovações de técnicas, produto, organizacionais e gerenciais - que incorporam um salto qualitativo na produtividade potencial para toda (ou a maioria) da economia e tornam acessível uma ampla gama incomum de oportunidades de investimento e lucro. Uma mudança em tal paradigma possui um efeito pervasivo sobre toda a economia, carregando consigo muitos clusters de inovações radicais e incrementais que podem, eventualmente, promover um conjunto de novos sistemas tecnológicos (FREEMAN; PEREZ, 1988).

\footnotetext{
${ }^{3}$ Segue-se esse Manual pois os dados da PINTEC, analisados na seção 5, são coletados seguindo esse Manual. Enquanto o conceito de Inovações Tecnológicas é mais homogêneo na literatura, a definição de inovações organizacionais é mais criticada na literatura (ARMBRUSTER et al., 2008), o que exigiu explicitar inicialmente qual o conceito utilizado no decorrer do artigo.
} 
Seguindo a abordagem de Freeman e Perez (1988), cada novo paradigma possui um conjunto de insumos tido como fatores-chave deste. Tais insumos devem preencher as seguintes condições: (i) custo relativo baixo e rapidamente decrescente; (ii) aparente disponibilidade quase ilimitada de oferta por longos períodos; e (iii) claro potencial para sua utilização em muitos produtos e processos ao longo do sistema econômico.

Tal fator-chave não surge isolado, mas sim conectado com crescentes e interligadas inovações técnicas, sociais e gerenciais, algumas relacionadas à produção do próprio fator central e outras à sua utilização. Um novo PTE reestrutura o sistema produtivo, envolvendo, entre outros pontos, (1) novas "melhores práticas" na organização da firma, (2) um novo mix de produtos intensivos no fator-chave; e (3) novas tendências de inovações radicais e incrementais em torno da difusão e uso mais intensivo do novo fator-chave (FREEMAN; PEREZ, 1988). Vale salientar que, seguindo as definições do Manual de Oslo, discutidas anteriormente, "novas práticas na organização da firma" podem se configurar como inovações organizacionais enquanto "um novo mix de produtos" pode se referir a inovações de produto.

Portanto, essa definição de Freeman e Perez (1988) quanto ao PTE pode ser reinterpretada dizendo que o desenvolvimento ou cristalização de um novo PTE envolve a realização tanto de inovações tecnológicas quanto inovações organizacionais que são inter-relacionadas, se retroalimentam e se dão em torno de um fator-chave, cujas características foram descritas anteriormente. Dois exemplos ilustram essa reinterpretação.

Os estudos de Chandler (1977) sobre a ascensão da grande empresa multidivisional norteamericana no início do século XX é um destes exemplos. Em sua perspectiva histórica, o autor mostra como o advento da ferrovia e do telégrafo - que podem ser entendidos como inovações tecnológicas relacionadas com a segunda onda de progresso técnico (FREEMAN; SOETE, 2008) ${ }^{4}$ - resultaram em novas formas de organizar e gerir as empresas. $\mathrm{O}$ autor destaca que as próprias empresas ligadas às ferrovias necessitaram de novas formas organizacionais para gerenciar tais inovações tecnológicas, sendo as primeiras a empregarem gestores em tempo integral para monitorar e coordenar diferentes unidades de produção. Chandler (1977) também destaca que as firmas que se utilizaram dessas novas tecnologias reorganizaram suas atividades produtivas em diferentes unidades e localidades, permitindo a criação e o desenvolvimento da firma multidivisional, a qual passou a integrar diversas atividades antes realizadas pelo mercado. Para Nelson (2006), o estudo de Chandler indica que a mudança organizacional seria um suporte ao progresso tecnológico.

Complementar ao estudo anterior, Nelson e Sampat (2001) sugerem que novas tecnologias físicas - e.g. novos produtos e novas máquinas - têm uma relação dupla com novas formas organizacionais $^{5}$ : de um lado, a implementação de uma nova tecnologia física só será eficaz se novas formas organizacionais (e.g. adoção de departamentos de P\&D e novas formas de treinamento) puderem se ajustar a elas, permitindo explora-las efetivamente. Por outro lado, a forma organizacional existente influencia a forma como a tecnologia física se expande. Os autores tratam especialmente dois exemplos. O primeiro, sobre o surgimento da produção em massa dos Estados Unidos, onde a adoção dessa nova forma de produção (i.e. uma inovação de processo) demandou novas formas organizacionais, como as descritas anteriormente por Chandler. Em segundo lugar, os autores analisam a indústria alemã de corantes sintéticos no fim do séc. XIX, início do séc. XX, mostrando como a criação de laboratórios de $\mathrm{P} \& \mathrm{D}$ dentro da empresa (i.e. inovações organizacionais) foram essenciais para gerar novos produtos baseados em avanços científicos quanto à estrutura de

\footnotetext{
${ }^{4}$ Freeman e Soete (2008) ilustram o conceito schumpeteriano de ondas longas baseadas em sucessivas transformações tecnológicas que se configurariam como paradigmas tecno-econômicos na visão de Freeman e Perez (1988). Os autores notam que Schumpeter descreveu tais ondas de progresso técnico como "revoluções industriais sucessivas", seguindo Nicolai Kondratieff na descrição destes ciclos longos, tidos pelos economistas como ondas ou fases de crescimento.

${ }^{5}$ Nelson e Sampat (2001) discutem a relação entre tecnologias sociais (e.g. treinamentos e formas organizacionais) e tecnologias físicas, como máquinas e equipamentos. As tecnologias sociais estabelecem uma estrutura de comportamento, abarcando, por exemplo, formas de educação e de organização da empresa. Assim, há certa semelhança entre os termos "inovação organizacional" e "tecnologia social", mas estes ainda não estão plenamente compatibilizados na literatura, porém, acredita-se que para os propósitos do presente estudo a interpretação realizada seja satisfatória.
} 
compostos orgânicos. Segundo os autores, esse novo método organizacional - laboratório de P\&D interno à firma - foi sendo difundido na economia na medida em que as firmas que o adotaram apresentaram maiores lucros.

Portanto, é possível destacar que as inovações tecnológicas e organizacionais caminham juntas e há uma retroalimentação entre elas: inovações organizacionais (IO) favorecem e são favorecidas pelas inovações tecnológicas (IT). Porém, os estudos anteriores têm um enfoque maior sobre a dimensão setorial ou no nível mais macro (do PTE) da relação entre IO e IT. Porém por que há essa retroalimentação entre IO e IT ao nível da firma em si? Isso é respondido na subseção abaixo.

\subsection{IO versus IT na dimensão da firma}

Para responder à questão anterior, duas abordagens podem ser utilizadas: (1) capacidades dinâmicas; (2) firma como um conjunto de rotinas.

Quanto às capacidades, a primeira relação aparece na definição de capacidades dinâmicas. Teece, Pisano e Shuen (1997) definem tal capacidade como as habilidades das empresas para integrar, construir e reconfigurar competências externas e internas para reagir rapidamente às mudanças no ambiente. Essas capacidades estão vinculadas a rotinas e processos organizacionais, que remetem às atividades realizadas através de ativos específicos da empresa reunidos em clusters integrados que se "espalham" entre indivíduos e grupos ${ }^{6}$ (TEECE; PISANO; SHUEN, 1997). Ou seja, caso a empresa busque inovar tecnologicamente como uma resposta a uma dada mudança no mercado, tal inovação dependerá de processos organizacionais internos e da posse de "ativos complementares" para se desenvolver e gerar resultados satisfatórios. Logo, o desenvolvimento de capacidades dinâmicas que permitirão à firma elevar suas capacidades em inovar tecnologicamente tende a exigir mudanças nos processos organizacionais que podem se configurar, portanto, como inovações organizacionais.

Ademais, a adoção de inovações organizacionais, especialmente no âmbito da organização do trabalho, pode contribuir para que a firma desenvolva maiores capacidades absortivas - $a$ la Cohen e Levinthal (1990) - na medida em que esta depende da forma como o conhecimento interno é gerado e difundido entre os trabalhadores (COHEN; LEVINTHAL, 1990; ZAHRA; GEORGE, 2002). Maiores capacidades de absorção permitem que a firma identifique melhores oportunidades externamente e tenham maiores chances de implementar inovações tecnológicas (MUROVEC; PRODAN, 2009).

Camisón e Villar-López (2014) complementam os dois argumentos ao identificarem, empiricamente, que a inovação organizacional favorece diretamente o desenvolvimento das capacidades de inovação de processo, mas contribui indiretamente para a capacidade de inovar em produto (mediado pela inovação de processo). Segundo os autores, a introdução de novas práticas organizacionais per se não conduz diretamente ao desenvolvimento de capacidade tecnológica para inovar em produto, pois essa relação depende de outros fatores organizacionais, como uma infraestrutura organizacional apropriada, habilidades tecnológicas e de engenharia para conceber os processos produtivos, o layout e a logística para dar suporte eficientemente à concepção de novos produtos e sua comercialização. Interpretando a partir de Teece, Pisano e Shuen (1997), a inovação organizacional precisa vir acompanhada do desenvolvimento de "ativos complementares" para favorecer a inovação de produto.

Por fim, pode-se remeter ao conceito de rotinas para explicar essa relação entre IO e IT no âmbito da firma, entendendo-as como mudanças nas rotinas das empresas. Segundo Nelson e Winter (2005), as rotinas são padrões de comportamento possíveis, abarcando tanto regras técnicas chamadas de características operacionais - relacionadas com os equipamentos e os fatores de produção existentes, quanto rotinas relacionadas às decisões de investimentos e à busca por melhorias nas características operacionais. As rotinas definem uma lista de funções que determinam como a

\footnotetext{
${ }^{6}$ A definição original é feita da seguinte forma: "When a firm-specific asset are assembled in integrated clusters spanning individuals and groups so that they enable distinctive activities to be performed, these activities constitute organizational routines and processes" (TEECE; PISANO; SHUEN, 1997, p. 516)
} 
firma age perante as condições externas (como de mercado) e internas, como estoque de maquinário ou taxa média de lucro de períodos anteriores. Assim, a execução de uma dada rotina depende do ambiente e também da organização interna da empresa, a qual afeta a transferência de conhecimentos e de informações entre os membros da empresa (NELSON; WINTER, 2005). Vromen (2006) vai além e afirma que as rotinas envolvem o modo como os membros da organização estão conectados funcionalmente e os artefatos ou tecnologias dos quais eles dependem. Para esse autor, as rotinas são padrões intra-organizacionais e de interações multi-pessoais que se repetem, sendo reveladas em um ambiente físico construído artificialmente ${ }^{7}$.

Portanto, pode-se argumentar que diferentes tecnologias (i.e. IT) e diferentes formas organizacionais (i.e. IO) estão atreladas a diferentes rotinas que se inter-relacionam. A título de exemplo, uma nova forma de organização dos trabalhadores, como a criação de um departamento de $\mathrm{P} \& \mathrm{D}$, poderia resultar na aplicação de distintas rotinas de busca que permitiriam identificar novas técnicas, aprimorando as características operacionais da firma, envolvendo outras "rotinas técnicas". Nesse sentido, o departamento de $\mathrm{P} \& \mathrm{D}$ promove uma reorganização das relações de trabalho na firma - se configurando como uma inovação organizacional - e utiliza distintas rotinas de busca. Essas rotinas serão mais efetivas para a empresa ao identificar novas tecnologias físicas, resultando, portanto em uma nova combinação de rotinas.

Se usarmos a noção de firma enquanto um sistema complexo (SIMON, 1996), onde seus elementos (i.e. suas rotinas) possuem relações epistáticas (i.e. mudanças em um elemento afeta o outo elemento) e podem ser combinados de diferentes formas (FRENKEN, 2006), pode-se dizer que mudanças nas rotinas decorrentes de inovações tecnológicas (ITs) tendem a ter maiores resultados (maiores fitness value) quando acompanhadas de inovações organizacionais (IOs) que alterem os processos organizacionais interligadas às ITs. Resultados empíricos reforçam essa "sinergia" entre as ITs e IOs, mostrando que elas são estratégias complementares (BATTISTI; STONEMAN, 2010) especialmente para grandes empresas (BALLOT et al. 2015) - e, quando combinadas, conferem maiores vantagens competitivas às firmas, aprimorando a qualidade e desempenho de produtos $\mathrm{e}$ serviços já existentes (EVANGELISTA; VEZZANI, 2010) e permitindo maior crescimento em termos de receita e emprego (SAPPRASERT; CLAUSEN, 2012).

Para exemplificar esse última relação, pode-se realizar uma releitura do trabalho de Kenaroglu (2003). Esse autor discute a importância de se alinhar o uso de tecnologias da informação com novas formas organizacionais para que as primeiras gerem um aumento de produtividade. $\mathrm{O}$ autor cita exemplos onde o efeito desejado da adoção de máquinas controladas por computadores e tecnologias de produção flexíveis só foi obtido a partir de alterações nas relações de trabalho na empresa em prol de formas de trabalho mais flexíveis, onde os trabalhadores da linha de produção passaram a ter maior autonomia e trabalhar em grupos. Em outras palavras, a adoção dessas novas tecnologias trouxe novas regras técnicas e exigiram novas formas de relação entre os trabalhadores, o que se pode configurar como uma nova combinação de rotinas com um fitness value superior ao que seria obtido se as novas tecnologias fossem adotadas sem as mudanças nas relações de trabalho.

Portanto, há evidências de que as inovações tecnológicas (ITs) e organizacionais (IOs) se retroalimentam e são uma "via de mão dupla": a realização de inovações tecnológicas tende a ser favorecida pelas inovações organizacionais - via, por exemplo, elevação das capacidades inovativas - ao mesmo tempo que as ITs podem demandar novas formas organizacionais (i.e. uma IO) para ter um efeito satisfatório para a firma obter vantagens competitivas via inovações tecnológicas. O trabalho de Kenaroglu (2003) indica que isso também é válido quando se discute a adoção de Tecnologias da Informação pelas firmas. A importância do setor desenvolvedor destas TI é discutido a seguir, focando para o caso de Minas Gerais.

\footnotetext{
${ }^{7}$ Para Simon (1996), um objeto é artificial quando, por ação humana, imita o natural, usando os mesmos materiais do objeto natural ou um pouco diferente.
} 


\section{3 - Tecnologia da Informação em Minas Gerais}

Em Minas Gerais, o setor de serviços é o que mais emprega pessoas e gera renda na economia. Em Minas Gerais, esse setor empregava cerca de 63,2\% das pessoas ocupadas em empregos formais (que somaram 4,92 milhões aproximadamente), de acordo com a RAIS de 2012. Sobre a renda gerada na economia, a média do valor adicionado bruto (VAB) para o setor de serviços como um todo é de mais de $60 \%$ do total do VAB mineiro entre 2010 e 2015. A Tabela 1 apresenta um panorama do emprego, de acordo com dados da RAIS 2012, e do VAB mineiro, de acordo com dados das Contas Regionais do Brasil, IBGE (2015).

Tabela 1 - Empregos Formais e Valor Adicionado Bruto (VAB) por setores - Minas Gerais

\begin{tabular}{l|c|c}
\hline Setores de Atividade Econômica & \% empregos formais 2012 & \% médio VAB (2010-2015) \\
\hline Agropecuária e Extrativas & 7.7 & 9.2 \\
Indústrias & 29.1 & 31.7 \\
Serviços & 63.2 & 63,5 \\
Total & $\mathbf{1 0 0 . 0}$ & $\mathbf{1 0 0 . 0}$ \\
\hline
\end{tabular}

Fonte: Elaboração a partir de RAIS (2012) e PIA-IBGE (2015).

A Tabela 2 apresenta algumas variáveis extraídas da Pesquisa Anual de Serviços (PAS) para o setor de serviços em Minas Gerais, para o ano de 2015. Observa-se concentração das variáveis Receita Bruta e remunerações geradas no setor de serviços em alguns setores. As remunerações se concentram marcadamente nas atividades de serviços profissionais, administrativos e complementares (com 37,7\% das remunerações do setor de serviços em Minas Gerais) e no setor de transportes e serviços auxiliares aos transportes e correio (com 27,9\%). No que se refere à receita bruta de prestação de serviços, os setores de serviços que mais se destacam são aqueles relacionados aos serviços de transportes e atividades afins (32,5\% da receita bruta total do setor se serviços do estado), serviços profissionais, administrativos e complementares $(25,6 \%)$, e os serviços de informação e comunicação (22\%).

Sobre o pessoal ocupado, as empresas de Minas Gerais entrevistadas pela PAS empregaram 1,17 milhões de pessoas, das quais $37,7 \%$ estavam no setor de serviços profissionais, administrativos e complementares, $23,0 \%$ nas empresas relacionadas às atividades de transportes e 24,6\% nas empresas que prestam serviços predominantemente às famílias. Como se verá nas seções seguintes deste trabalho, entre as atividades de serviços que mais inovam estão as de informação. Essas atividades empregam proporcionalmente menos pessoas e as remunerações correspondentes são menores que as de outros segmentos de serviços.

Sobre o indicador que relaciona as remunerações do capital humano à receita bruta da empresa, é necessário salientar que esse foi o indicador disponível mais próximo para medir a participação da renda do trabalho nas atividades de serviços. O mais adequado seria medir a participação dessa renda frente ao valor adicionado obtido no ano para cada segmento do setor de serviços, mas não foi possível obter esses valores no nível regional pela PAS. Os segmentos do setor de serviços que apresentam maior participação das remunerações na receita bruta das empresas são os de manutenção e reparação $(41,1 \%)$, os de atividades profissionais, administrativas e complementares $(30,4 \%)$ e de serviços prestados principalmente às famílias $(25,5 \%)$. Finalmente, é importante verificar que a participação das remunerações no segmento de serviços de informação e comunicação é baixa dentre os demais segmentos de serviços relacionados na PAS $(10,7 \%)$. 
Tabela 2 - Percentuais das receitas brutas, massas salariais e pessoal ocupado, e proporção das remunerações na receita bruta. Empresas de serviços. Minas Gerais, 2015.

\begin{tabular}{|c|c|c|c|c|c|}
\hline Minas Gerais & $\begin{array}{l}\% \text { Receita } \\
\text { Bruta de } \\
\text { prestação de } \\
\text { serviços }\end{array}$ & $\begin{array}{c}\text { \% dos } \\
\text { salários, } \\
\text { retiradas e } \\
\text { outras } \\
\text { remunerações }\end{array}$ & $\begin{array}{c}\% \text { do PO } \\
(\text { em } 31 / 12)\end{array}$ & $\begin{array}{l}\text { Remunerações/ } \\
\text { receita bruta de } \\
\text { prestação de } \\
\text { serviços }(\%)\end{array}$ & $\begin{array}{c}\text { Número } \\
\text { médio de } \\
\text { empregados } \\
\text { por } \\
\text { empresa }(*)\end{array}$ \\
\hline Minas Gerais - Total & 100,0 & 100,0 & 100,0 & $100,0 \%$ & 8 \\
\hline Serviços prestados principalmente às famílias & 12,9 & 16,0 & 24,6 & $25,5 \%$ & 6 , \\
\hline Serviços de informação e comunicação & 22,0 & 11,5 & 6,6 & $10,7 \%$ & 8 \\
\hline $\begin{array}{l}\text { Serviços profissionais, administrativos e } \\
\text { complementares }\end{array}$ & 25,6 & 37,7 & 37,0 & $30,4 \%$ & 10 \\
\hline $\begin{array}{l}\text { Transportes, serviços auxiliares aos } \\
\text { transportes e correio }\end{array}$ & 32,5 & 27,9 & 23,0 & $17,7 \%$ & 13 \\
\hline Atividades imobiliárias & 2,1 & 0,9 & 1,6 & $9,3 \%$ & 3 \\
\hline Serviços de manutenção e reparação & 1,5 & 3,0 & 4,4 & $41,1 \%$ & 4 \\
\hline Outras atividades de serviços & 34 & 3,0 & 2,8 & $17,9 \%$ & 7 \\
\hline
\end{tabular}

Obs.: * Arredondado para obter o número inteiro de empregados.

Fonte: Elaboração própria a partir de PAS (2012)

O setor de Serviços de Informação e Comunicação foram responsáveis, portanto, por $22 \%$ do total da receita bruta do setor de Serviços em Minas Gerais em 2015. De acordo com informações do site do $\mathrm{INDI}^{8}$, em Minas Gerais existem cerca de 5 mil empresas, com faturamento anual de R $\$ 2,3$ bilhões, gerando cerca de 33 mil empregos diretos. Deste conjunto, 70\% das empresas estão localizadas em Belo Horizonte e na Região Metropolitana.

Também de acordo com o INDI, na área de hardware em Minas Gerais estão localizadas duas empresas de Data Centers. A Ativas que possui infraestrutura que segue os mais modernos parâmetros internacionais de sustentabilidade ambiental e eficiência energética, localizada em Belo Horizonte e a Algar Tecnologia, em Uberlândia, com dois Data Centers. E também é importante mencionar que a RMBH abriga importantes empresas do setor como o Google e o seu Centro de P\&D (único na América Latina), a Microsoft, SAP, Algar Tech, Avenue Code, Infosys, Sankhya e Totvs.

Por sua vez, na área de software, Belo Horizonte possui um polo tecnológico importante de destaque no cenário nacional. De acordo com Vieira et. al (2007), dentre as instituições relevantes na governança do polo estão a Fumsoft, Assespro-MG e o Sindinfor-MG. As atividades realizadas por estas instituições abrangem: projetos de pré-incubação e incubação de empresas (Incubadora Insoft), treinamento, capacitação em gestão empresarial, assistência jurídica, fornecimento de infraestrutura e instalações para realização de reuniões, palestras e conferências. Ademais, pode-se citar também o agendamento de participação em feiras, exposições e seminários de interesse das empresas; e o apoio estratégico e institucional para obtenção de linhas de financiamento, para participação em projetos conjuntos e para participação em Rodas de Negócios e importantes eventos da área (VIEIRA et. al. 2007:31).

Dentre os Programas da FUMSOFT destaca-se o Programa de aceleração o ACELERA MGTI que tem como objetivo o desenvolvimento de empresas de base tecnológica. Desde 2014, o Programa é operacionalizado em conjunto com a Universidade de Stanford, no Vale do Silício. Desde a sua criação, em 1996, o ACELERA MGTI já desenvolveu 56 empresas e atualmente (2018) está desenvolvendo 9 empresas.

O Setor de Tecnologia de Informação e de Comunicação TIC) vem sendo alvo de políticas industriais e de inovação há cerca de uma década por parte do Governo Federal e mais recentemente por parte do governo Estadual. Isto implica que há recursos crescentes sendo destinados a atividades

\footnotetext{
${ }^{8}$ http://www.indi.mg.gov.br/
} 
de inovação para este setor. Por exemplo, entre 2006 e 2015, 16 empresas mineiras do Setor de TI receberam incentivos fiscais, sendo que a metade conseguiu incentivos em mais de um ano.

\section{4 - Análise empírica}

A presente seção traz evidências empíricas sobre a relação entre inovação organizacional e tecnológica, focando no setor de Tecnologias da Informação e comparando os resultados ao nível Brasil e Minas Gerais. Primeiramente são apresentadas questões metodológicos sobre a PINTEC e sua capacidade em prover informações sobre o fenômeno proposto. Em seguida, são discutidos os dados.

\subsection{PINTEC: Aspectos Metodológicos}

A Pesquisa de Inovação Tecnológica (PINTEC), realizada pelo IBGE desde 2000, reúne informações sobre as atividades inovativas realizadas pelas empresas industriais brasileiras. A pesquisa foi construída a partir da concepção da inovação como um processo interativo, abrangendo a interdependência entre os diversos atores, e o ambiente institucional e econômico no qual estão inseridos. A definição de empresa inovadora é bastante ampla, considerando tanto a empresa que desenvolveu sua inovação como a que adotou uma inovação gerada por outra empresa. Após este passo, a empresa inovadora responde a um conjunto de perguntas que visam inferir os esforços, os fatores que influenciam, bem como os impactos da inovação.

No seu conjunto a PINTEC apresenta informações sobre atividades inovativas realizadas pelas empresas abrangendo: (a) dispêndios com as atividades inovativas; (b) fontes de financiamento dos gastos; (c) impacto das inovações no desempenho das empresas; (d) fontes de informação utilizadas; (e) arranjos cooperativos estabelecidos; (f) papel dos incentivos governamentais; (g) obstáculos às atividades de inovação; h) patentes e outros métodos de proteção; i) realização de inovações organizacionais elou de marketing. As informações são disponibilizadas para o Brasil, Grandes Regiões e Unidades da Federação, permitindo, portanto, analisar a atividade inovativa em Minas Gerais. Para alguns setores os dados são disponibilizados, sendo também possível a análise ao nível setorial e estadual.

Até o momento o IBGE já realizou seis levantamentos, sendo referentes aos períodos de 19982000 (PINTEC 2000); 2001-2003 (PINTEC 2003), 2003-2005 (PINTEC 2005), 2006-2008 (PINTEC 2008), 2009-2011 (PINTEC 2011) e 2012-2014 (PINTEC, 2014). Em 2005 a PINTEC incluiu o setor de serviços ${ }^{9}$, sendo então, fornecidas as primeiras evidências estatísticas sobre a inovação tecnológica em serviços. A cada edição vem sendo ampliado o número de setores de serviços cobertos pela pesquisa $^{10}$. As seis pesquisas permitem, pois, caracterizar a dinâmica inovativa em mais de uma década.

A Pesquisa de Inovação (PINTEC) segue as recomendações do Manual de Oslo, definindo a inovação tecnológica como a implementação de produtos (bens ou serviços) ou processos tecnologicamente novos ou substancialmente aprimorados. Produto tecnologicamente novo é aquele "cujas características fundamentais (especificações técnicas, usos pretendidos, software ou outro componente imaterial incorporado) diferem significativamente de todos os produtos previamente produzidos pela empresa" (IBGE,2003:20). Por sua vez, a inovação tecnológica de processo referese "ao processo tecnologicamente novo ou substancialmente aprimorado, que envolve a introdução de tecnologia de produção nova ou significativamente aperfeiçoada, assim como de métodos novos

\footnotetext{
${ }^{9}$ A ampliação do universo de investigação para incorporar, além das atividades das indústrias extrativas e de transformação, os serviços de alta intensidade tecnológica justificou a alteração no título da pesquisa, anteriormente denominada Pesquisa Industrial de Inovação Tecnológica, para Pesquisa de Inovação Tecnológica (IBGE, 2007). Por sua vez, o termo "tecnológica" foi suprimido do nome da pesquisa na edição de 2011 uma vez que passou a mensurar também a inovação organizacional.

${ }^{10}$ A PINTEC 2005 contemplava os setores de telecomunicações, atividades de informática e serviços relacionados e pesquisa e desenvolvimento (P\&D). A PINTEC 2008 incorporou os setores de 'edição e gravação e edição de música' e 'tratamento de dados, hospedagem na Internet e outras atividades relacionadas' e na pesquisa de 2011 foram inseridos os "serviços de arquitetura e engenharia, testes e análises técnicas".
} 
ou substancialmente aprimorados para manuseio e entrega de produtos (acondicionamento e preservação)" (IBGE, 2003:21). É importante ressaltar que a inovação tecnológica de produto e/ou processo novo refere-se à empresa, não sendo necessariamente novo para o mercado e/ou setor de atuação.

Por sua vez, a inovação organizacional "compreende a implementação de novas técnicas de gestão ou de significativas mudanças na organização do trabalho e nas relações externas da empresa, com vistas a melhorar o uso do conhecimento, a eficiência dos fluxos de trabalho ou a qualidade dos bens ou serviços. Deve ser resultado de decisões estratégicas tomadas pela direção e constituir novidade organizativa para a empresa" (IBGE, 2014:11). Não são consideradas fusões e aquisições. A inovação de marketing "é a implementação de novas estratégias ou conceitos de marketing que diferem significativamente dos usados previamente pela empresa. Supõe mudanças significativas no desenho ou embalagem do produto, nos seus canais de venda, em sua promoção ou na fixação de preços, sem modificar as características funcionais ou de uso do produto. Visam abrir novos mercados ou reposicionar o produto no mercado" (IBGE, 2014:11).

A próxima seção apresenta os dados para Minas Gerais e para o Brasil do setor de Tecnologia da Informação e da Comunicação (TIC) referentes a esforço inovador, inovação organizacional e tecnológica e à rigidez organizacional dentre os obstáculos à inovação. Foram analisadas as informações do setor "Atividades dos serviços de tecnologia da informação" para o Brasil e Minas Gerais nas pesquisas de 2009-2011 e 2012-2014.

\section{4- Inovação tecnológica e organizacional nos setores de Tecnologia da Informação em Minas Gerais e no Brasil}

Há um aumento no número de empresas que inovaram em todos os tipos, conforme evidenciado na tabela 3. Em torno de $45 \%$ das empresas de TI implementaram inovações no Brasil tanto no período 2009-2011, quanto no período 2012-2014. Para Minas Gerais é possível verificar uma queda no percentual de empresas que implementaram inovação: de 46,5\% em 2011 para 31,5\% em 2014. Essa queda, porém, é fruto do aumento do número total de empresas de TI no Estado, visto que o valor total de empresas que implementaram inovação aumentou (de 131 empresas para 168 empresas). Esse resultado é alcançado tanto por um aumento no número de empresas que implementaram inovação de produto quanto por empresas que implementaram inovação de processo. Aliás, a comparação percentual mostra que as empresas de TI de Minas Gerais são mais inovadoras que a média brasileira para esses dois tipos de inovação.

O grau de novidade também é uma observação interessante de ser feita. Pode-se verificar que tanto no Brasil quanto em Minas Gerais há uma diminuição no percentual de empresas que implementam inovação que são novas apenas nos seus limites de produção e/ou de processo ("novo para a empresa'). No outro grau de novidade (produto ou processo novo para o mercado nacional) o comportamento é dispare. Enquanto para o Brasil há uma queda na inovação de produto novo para o mercado nacional e um comportamento estável na inovação de processo para o mercado nacional, o percentual em Minas Gerais cresce substancialmente tanto para inovação de produto quanto de processo; para produto, mais do que dobra (22\% para $45 \%$ ) e para processo cresce $567 \%$ (6\% para $40 \%$ ). 
Tabela 3 - Empresas, total e as que implementaram inovações e/ou com projetos, serviços de tecnologia da informação - Brasil / Minas Gerais - período 2011 - 2014

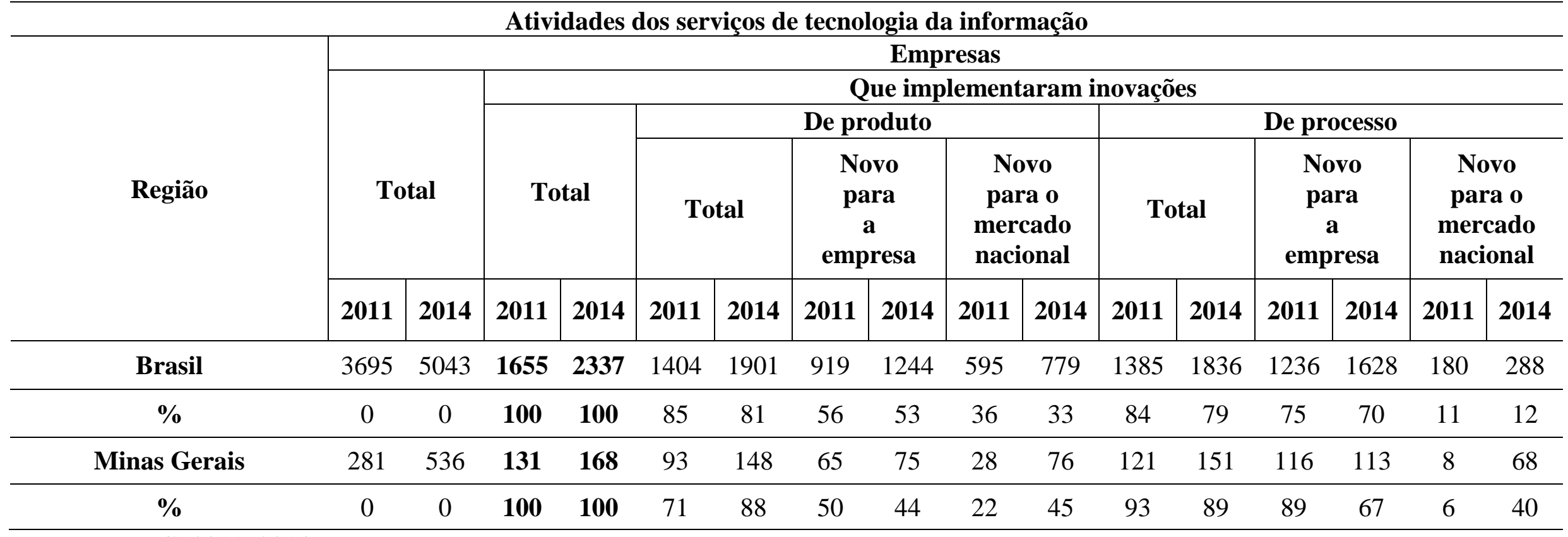

Fonte: PINTEC (2011, 2014). 
$\mathrm{Na}$ Tabela 4 são apresentadas informações sobre o total de empresas que implementaram inovação e, dentre essas, aquelas que possuem atividades internas de pesquisa e desenvolvimento (P\&D). Novamente podem ser observadas diferenças consideráveis na comparação entre Brasil e Minas Gerais. A despeito do crescimento observado para ambos recortes territoriais, Minas Gerais possui maior percentual de empresas com atividades internas de P\&D. Esse percentual sofre uma queda no período observado (Brasil e Minas Gerais), mas os melhores resultados para Minas Gerais ainda são consideráveis. Este resultado pode ser explicado pelo polo de TIC existente na RMBH e também pela presença de empresas de grande porte e consolidadas no setor localizadas no Estado, conforme mencionado na Seção $3^{11}$.

Tabela 4 - Empresas, total e as que implementaram inovações, serviços de tecnologia da informação - Brasil / Minas Gerais - período 2011 - 2014

Atividades dos serviços de tecnologia da informação

\begin{tabular}{c|c|c|c|c}
\hline \multirow{2}{*}{ Região } & \multicolumn{4}{c}{$\begin{array}{c}\text { Empresas que implementaram } \\
\text { inovações }\end{array}$} \\
\cline { 2 - 5 } & \multicolumn{2}{|c}{ Total } & \multicolumn{2}{c}{$\begin{array}{c}\text { Atividades internas } \\
\text { de P\&D }\end{array}$} \\
\cline { 2 - 5 } & $\mathbf{2 0 1 1}$ & $\mathbf{2 0 1 4}$ & $\mathbf{2 0 1 1}$ & $\mathbf{2 0 1 4}$ \\
\hline Brasil & $\mathbf{1 6 5 5}$ & $\mathbf{2 3 3 7}$ & 953 & 1265 \\
\hline$\%$ & $\mathbf{1 0 0}$ & $\mathbf{1 0 0}$ & 58 & 54 \\
\hline $\begin{array}{c}\text { Minas } \\
\text { Gerais }\end{array}$ & $\mathbf{1 3 1}$ & $\mathbf{1 6 8}$ & 121 & 139 \\
\hline \% & $\mathbf{1 0 0}$ & $\mathbf{1 0 0}$ & 93 & 82 \\
\hline
\end{tabular}

Fonte: PINTEC (2011, 2014).

A observação da Tabela 5 amplia o entendimento das diferenças territoriais, quando observado o grau de importância atribuído pela empresa às atividades internas de P\&D; empresas de TI de Minas Gerais atribuem percentual consistentemente superior para 'alta importância' quando comparado com a média brasileira. Mesmo com uma queda desse percentual de 2011 para 2014, os resultados ainda são superiores.

Ainda, a Tabela 5 nos permite fazer juízo de valor quanto à diferença do setor de serviços de TI em relação à média do setor de serviços como um todo. Em suma, a importância de atividades internas de $\mathrm{P} \& \mathrm{D}$ é maior para esse tipo de serviço, o que já era esperado visto a natureza da qualificação e sofisticação das atividades.

Tabela 5 - Grau de importância atribuída às atividades internas de P\&D - Empresas, total e as que implementaram inovações, serviços de tecnologia da informação - Brasil / Minas

Gerais - período 2011 - 2014

\begin{tabular}{l|l|c|c|c|c|c}
\hline \multirow{2}{*}{ Anos } & \multirow{2}{*}{ Descrição } & \multirow{2}{*}{ Total } & \multicolumn{4}{|c}{ Grau de importância } \\
\cline { 4 - 7 } & & & Alta & \% alta & Média & \% média \\
\hline \multirow{3}{*}{$\mathbf{2 0 1 4}$} & Serviços - Brasil & $\mathbf{4 5 6 9}$ & $\mathbf{1 3 8 3}$ & $\mathbf{3 0}$ & $\mathbf{4 0 9}$ & $\mathbf{9}$ \\
& Serviços de TI & 2337 & 971 & 42 & 294 & 13 \\
& Serviços - MG & $\mathbf{4 5 9}$ & $\mathbf{1 2 8}$ & $\mathbf{2 8}$ & $\mathbf{5 0}$ & $\mathbf{1 1}$ \\
& Serviços de TI & 168 & 110 & 66 & 28 & 17 \\
\hline
\end{tabular}

\footnotetext{
11 E importante recordar que considera-se como empresa localizada em Minas Gerais apenas a empresa com sede no Estado, de forma, que, por exemplo, se uma empresa tem sede em São Paulo e seu centro de P\&D fica em Minas Gerais, todo o P\&D feito por esta empresas contabiliza em São Paulo.
} 


\begin{tabular}{l|lccccc}
$\mathbf{2 0 1 1}$ & Serviços - Brasil & $\mathbf{4 2 5 8}$ & $\mathbf{8 4 0}$ & $\mathbf{2 0}$ & $\mathbf{6 9 9}$ & $\mathbf{1 6}$ \\
& Serviços de TI & 1655 & 591 & 36 & 363 & 22 \\
Serviços - MG & $\mathbf{4 1 7}$ & $\mathbf{1 2 5}$ & $\mathbf{3 0}$ & $\mathbf{4 5}$ & $\mathbf{1 1}$ \\
& Serviços de TI & 131 & 111 & 85 & 10 & 8 \\
\hline \multicolumn{7}{c}{ Fonte: PINTEC $(2011,2014)}$.
\end{tabular}

Importante ainda é a observação da evolução da introdução de inovações organizacionais e de marketing nas empresas de TI. A tabela 6 apresenta a adoção de inovações organizacionais e seus tipos para as empresas que também realizaram inovações tecnológicas (que apresentaram inovação de produtos e processos) e para as que não realizaram inovação tecnológica.

Mesmo não tendo implementado inovação de produtos, serviços ou ter algum projeto de inovação, uma quantidade considerável de empresas do setor de serviços de TI se engajou em inovações organizacionais ou de marketing. As principais estratégias de inovações desse tipo foram em técnicas de gestão, organização do trabalho e conceitos/estratégias de marketing. Mais uma vez os resultados de Minas Gerais são emblemáticos; 72\% dessas empresas implementaram inovação organizacional em técnicas de gestão e $82 \%$ implementaram inovação na organização do trabalho em 2014, números superiores aos observados em 2011. Além disso, inovações em conceitos/estratégias de marketing foram perseguidas por $75 \%$ das empresas em 2014.

Tal comportamento (aumento do percentual de empresas entre as duas pesquisas) segue uma evolução distinta da observada na média brasileira, onde é verificada uma queda ou pequeno crescimento (em termos percentuais) para qualquer um dos tipos de inovação organizacional ou de marketing analisados. Na organização do trabalho (inovação organizacional) e em estética, desenhos ou outras mudanças (inovação de marketing) houve uma redução no caso Brasileiro. Em suma, isso indica que o comportamento das empresas mineiras menos inovadoras no setor de TI foi dispare à média das empresas nacionais no período. Mesmo que tais empresas não possuam capacitação competitiva focada na diferenciação de produtos e/ou processos, elas procuraram estabelecer novas estratégias organizacionais e de marketing para se posicionar frente a modificações nos padrões concorrenciais. Portanto, é possível que exista em Minas Gerais uma parcela de empresas mineiras que realizam inovações organizacionais de modo substitutivo e não complementar às inovações tecnológicas. Estudos futuros podem comparar as vantagens competitivas entre as empresas mineiras que combinam tais inovações vis a vis a que os substituem. 
Tabela 6 - Empresas, total e as que não implementaram inovações, com indicação das inovações organizacionais e/ou de marketing implementadas, segundo as atividades dos serviços de tecnologia da informação - Brasil / Minas Gerais - período 2011 - 2014

\begin{tabular}{|c|c|c|c|c|c|c|c|c|c|c|c|c|c|c|c|c|}
\hline \multirow{4}{*}{$\begin{array}{l}\text { Tipos de } \\
\text { Inovação }\end{array}$} & \multirow{4}{*}{ Região } & \multirow{3}{*}{\multicolumn{2}{|c|}{ Total }} & \multicolumn{13}{|c|}{ Empresas } \\
\hline & & & & \multirow{2}{*}{\multicolumn{2}{|c|}{$\begin{array}{c}\text { Total } \\
\text { (Tipo de } \\
\text { inovação) }\end{array}$}} & \multicolumn{8}{|c|}{ Inovações organizacionais } & \multicolumn{3}{|c|}{ Inovações de marketil } \\
\hline & & & & & & \multicolumn{2}{|c|}{$\begin{array}{l}\text { Técnicas de } \\
\text { gestão }\end{array}$} & \multicolumn{2}{|c|}{$\begin{array}{c}\text { Técnicas de } \\
\text { gestão } \\
\text { ambiental }\end{array}$} & \multicolumn{2}{|c|}{$\begin{array}{c}\text { Organização do } \\
\text { trabalho }\end{array}$} & \multicolumn{2}{|c|}{$\begin{array}{l}\text { Relações } \\
\text { externas }\end{array}$} & \multicolumn{2}{|c|}{$\begin{array}{c}\text { Conceitos/estratégias } \\
\text { de marketing }\end{array}$} & \multirow{2}{*}{$\begin{array}{r}\begin{array}{r}\text { Estética, } \\
\text { outras 1 }\end{array} \\
2011\end{array}$} \\
\hline & & 2011 & 2014 & 2011 & 2014 & 2011 & 2014 & 2011 & 2014 & 2011 & 2014 & 2011 & 2014 & 2011 & 2014 & \\
\hline \multirow{4}{*}{$\begin{array}{c}\text { Que não } \\
\text { implementaram } \\
\text { produtos ou } \\
\text { processo sem } \\
\text { projeto }\end{array}$} & Brasil & 3695 & 5043 & 1887 & 2598 & 675 & 995 & 35 & 64 & 782 & 867 & 192 & 366 & 413 & 734 & 317 \\
\hline & $\%$ & 0 & 0 & 100 & 100 & 36 & 38 & 2 & 2 & 41 & 33 & 10 & 14 & 22 & 28 & 17 \\
\hline & $\begin{array}{l}\text { Minas } \\
\text { Gerais }\end{array}$ & 281 & 536 & 151 & 363 & 102 & 260 & 0 & 9 & 91 & 300 & 44 & 4 & 68 & 274 & 44 \\
\hline & $\%$ & 0 & 0 & 100 & 100 & 68 & 72 & 0 & 3 & 60 & 83 & 30 & 1 & 45 & 75 & 30 \\
\hline \multirow{4}{*}{\begin{tabular}{|c|} 
Que \\
implementaram \\
produto ou \\
processo sem e \\
sem projetos
\end{tabular}} & Brasil & 3695 & 5043 & 1655 & 2337 & 1251 & 1770 & 281 & 107 & 1241 & 1931 & 701 & 818 & 717 & 1259 & 680 \\
\hline & $\%$ & 0 & 0 & 100 & 100 & 76 & 76 & 17 & 5 & 75 & 83 & 42 & 35 & 43 & 54 & 41 \\
\hline & $\begin{array}{l}\text { Minas } \\
\text { Gerais }\end{array}$ & 281 & 536 & 131 & 168 & 75 & 150 & 16 & 23 & 74 & 148 & 105 & 47 & 103 & 100 & 95 \\
\hline & $\%$ & 0 & 0 & 100 & 100 & 58 & 89 & 12 & 14 & 56 & 88 & 80 & 28 & 79 & 59 & 73 \\
\hline
\end{tabular}

Fonte: PINTEC $(2011,2014)$. 
Comportamento parecido é verificado para as empresas mineiras que estabeleceram inovações de produto e/ou processo, conforme também apresentado na Tabela 6. Nesse caso, as inovações organizacionais 'técnicas de gestão' e 'organização do trabalho' destacam-se com o aumento percentual considerável de empresas (de $58 \%$ para $89 \%$ e $56 \%$ para $88 \%$, respectivamente), mas agora com queda de importância da estratégia de marketing 'conceitos/estratégias de marketing', seguindo padrão distinto do brasileiro que apresentou aumento entre as pesquisas de 2009-2011 e 2012-2014.

Por fim, é realizado a seguir uma análise sobre a importância dos problemas e obstáculos relacionados à rigidez organizacional para as empresas, diferenciando entre aquelas que não implementaram e as que implementaram inovação (Tabela 7). Os valores estão relacionados a empresas que declararam 'média importância' e 'alta importância' para tal tipo de problema. O número de empresas na tabela é diferente do apresentado em tabelas anteriores porque nem todas as empresas respondem às perguntas em relação aos problemas e obstáculos.

Tabela 7 - Empresas, total, as que implementaram e as que não implementaram inovações, por grau de importância dos problemas e obstáculos apontados, segundo as atividades dos serviços de tecnologia da informação - Brasil / Minas Gerais - período 2011 - 2014

\begin{tabular}{|c|c|c|c|c|c|c|c|}
\hline \multirow{4}{*}{$\begin{array}{c}\text { Inovação } \\
\text { Tecnológica }\end{array}$} & \multirow{4}{*}{ Região } & \multicolumn{6}{|c|}{ Empresas } \\
\hline & & \multirow{2}{*}{\multicolumn{2}{|c|}{$\begin{array}{c}\text { Total } \\
(\text { amostra) }\end{array}$}} & \multirow{2}{*}{\multicolumn{2}{|c|}{$\begin{array}{c}\text { Total } \\
\text { (responderam } \\
\text { à pergunta) }\end{array}$}} & \multirow{2}{*}{\multicolumn{2}{|c|}{$\begin{array}{c}\text { Grau de importância } \\
\text { dos problemas e } \\
\text { obstáculos apontados }\end{array}$}} \\
\hline & & & & & & & \\
\hline & & 2011 & 2014 & 2011 & 2014 & 2011 & 2014 \\
\hline \multirow{4}{*}{$\begin{array}{l}\text { Que não } \\
\text { inovaram }\end{array}$} & Brasil & 3695 & 5043 & 711 & 679 & 178 & 138 \\
\hline & $\%$ & 0 & 0 & 100 & 100 & 25 & 20 \\
\hline & Minas Gerais & 281 & 536 & 71 & 48 & 3 & 16 \\
\hline & $\%$ & 0 & 0 & 100 & 100 & 4 & 33 \\
\hline \multirow{4}{*}{$\begin{array}{c}\text { Que } \\
\text { inovaram }\end{array}$} & Brasil & 3695 & 5043 & 906 & 1600 & 422 & 572 \\
\hline & $\%$ & 0 & 0 & 100 & 100 & 47 & 36 \\
\hline & Minas Gerais & 281 & 536 & 107 & 120 & 74 & 12 \\
\hline & $\%$ & 0 & 0 & 100 & 100 & 69 & 10 \\
\hline
\end{tabular}

De qualquer forma, os resultados encontrados mostram que há uma queda de importância dos problemas e obstáculos advindos de rigidez organizacional para empresas que inovaram, e aumento, para o caso de Minas Gerais, em empresas que não inovaram tecnologicamente. Esse resultado pode estar ligado à adoção de inovações organizacionais em conjunto com a implementação de inovações tecnológicas, conforme verificado na Tabela 6.

\section{6 - Conclusões}

O arcabouço teórico desenvolvido neste artigo aponta para a importante articulação entre inovação tecnológica e organizacional ao nível meso-macro, ou de paradigmas tecno-econômicos, e também ao nível da firma. A interdependência entre estes dois tipos de inovação, ainda que relevante e já destacado por importantes estudiosos da mudança tecnológica (CHANDLER, 1977, NELSON, 2006; NELSON; SAMPAT, , 2001), tem sido pouco estudada e analisada na perspectiva da Economia. Neste sentido, este trabalho teve como objetivo avançar na articulação teórica entre estes 
dois tipos de inovação, entendendo ser um passo importante no avanço da compreensão sobre os determinantes intra-firma do desempenho inovativo (PARANHOS; HASENCLEVER, 2017).

O presente estudo tem como foco o setor de serviços ligados às Tecnologias da Informação no estado de Minas Gerais. Isso se justifica, pois, o presente setor está no cerne do atual paradigma tecno-econômico e tem ganhado espaço na economia de Minas Gerais. Ademais, o setor de serviços caracteriza-se pela grande relevância da inovação organizacional vis-à-vis na indústria de transformação. Ademais, esse setor. Foram analisados dados de duas edições da Pesquisa de Inovação (PINTEC), anos nos quais foi possível uma comparação ao nível setorial e regional, com foco no Estado de Minas Gerais.

Os dados analisados apontam um aumento no número de empresas de serviços de tecnologia da informação que inovaram, tanto para o Brasil quanto para o Estado de Minas Gerais. É verdade que para o último (MG) há uma queda percentual, mas isso é fruto da entrada no mercado de novas empresas de TI na amostra da PINTEC. Aliás, os dados apontam vantagem para Minas Gerais na comparação com o Brasil, tanto para inovação de produto quanto para inovação de processo.

Além de ser mais inovadoras, as empresas de MG apresentaram evolução na introdução de produtos e processos com maior grau de novidade, notadamente para o mercado nacional, diferentemente dos resultados para a amostra brasileira. Esse resultado caminha conjuntamente ao elevado percentual de empresas mineiras com atividades internas de $\mathrm{P} \& \mathrm{D}$, que pode ser explicado pela existência de um polo de TIC na RMBH e na presença de importantes empresas do setor no Estado.

Mesmo quando analisadas informações de inovação organizacional e de marketing, resultados satisfatórios para empresas mineiras são encontrados, tanto para as inovadoras quanto para as que não inovaram. Para ambos os tipos, inovações organizacionais ligadas à gestão e à organização do trabalho mostraram-se importantes, diferentemente do caso brasileiro. A diferença nessa observação talvez esteja ligada à eficiência das empresas em relação à inovação organizacional. Enquanto para empresas inovadoras parece que a inovação organizacional reduziu a importância de problemas de rigidez organizacional, para as empresas que não inovaram o resultado foi contrário. Há aqui um indício da relevância de uma associação entre inovação de produto/processo e inovação organizacional para melhorias no âmbito gerencial de empresas de TI.

Em suma, o setor de TI de Minas Gerais parece ter caminhado, no período analisado, para uma consolidação das capacidades de inovação, com aumento da sofisticação de produtos e processos, com grande importância das atividades de P\&D e inovações ligadas ao ambiente organizacional. Essa diferenciação de estratégia em relação às empresas brasileiras pode ter sido um diferencial competitivo, até um catalisador para o aumento do número de empresas de TI em MG na amostra da PINTEC (em torno de $90 \%$ ), o que pode ser resultado das políticas federais e estaduais de suporte ao setor no período recente. O desafio que se põe, portanto, é criar as condições e incentivos necessários para que essas empresas nascentes, que provavelmente ainda não possuem estratégias de inovação bem definidas, sigam o comportamento das empresas incumbentes do mercado, aumentando o percentual de empresas inovadoras.

\section{REFERENCIAS}

ARMBRUSTER, $\mathrm{H}$. et al. Organizational innovation: The challenge of measuring non-technical innovation in large-scale surveys. Technovation, v. 28, n. 10, p. 644-657, 2008.

BALLOT, G.; FAKHFAKH, F.; GALIA, F.; SALTER, A. The fateful triangle: Complementarities in performance between product, process and organizational innovation in France and the UK. Research Policy, v.44, 2015, p. 217-232. 
BATTISTI, G.; STONEMAN, P. How Innovative are UK Firms? Evidence from the Fourth UK Community Innovation Survey on Synergies between Technological and Organizational Innovations. British Journal of Management, vol. 21, 187-206, 2010.

CAMISÓN, C.; VILLAR-LÓPEZ, A. Organizational innovation as an enabler of technological innovation capabilities and firm performance. Journal of Bussiness Research, 67, p. 2891-2902, 2014.

CHANDlER, A. D. The Visible Hand: The Managerial Revolution in American Business. Massachusetts: The Belknap Press of Harvard University Press, 1977.

COHEN, W. M.; LEVINTHAL, D. A. Absorptive Capacity: A New Perspective on Learning and Innovation. Administrative Science Quarterly, v. 35, p. 128-152, 1990.

EVANGELISTA, R.; VEZZANI, A. The economic impact of technological and organizational innovations. A firm-level analysis. Research Policy, v.39, 2010, p. 1253-1263.

FREEMAN, C.; PEREZ, C. Structural crises of adjustment, business cycles and investment behaviour. In: DOSI, G. et al. Technical change and economic theory. Pinter Publishers, London, NY, 1988, pp. 38-66.

FREEMAN, C.; SOETE, L. A economia da inovação industrial. Campinas: Editora da Unicamp, 2008.

FRENKEN, K. Innovation, Evolution and Complexity Theory. 1. ed. Northampton: Edward Elgar Publishing, 2006.

IBGE Pesquisa de Inovação. 2003.

IBGE. Pesquisa de Inovação. 2014, Questionário.

KENAROGLU, B. Implications of Information Technology in Developing Countries and Its Impact in Organizational Change: STPS Working Papers, n. 302 Çankaya, Turquia.

KON, A. Economia de serviços: teoria e evolução no Brasil. Rio de Janeiro: Elsevier, 2004.

KUBOTA, L. C. A contribuição dos SICs para a inovação tecnológica das firmas de serviços. In: BERNARDES, R.; ANDREASSI, T. (Orgs) Inovação em serviços intensivos em conhecimento. São Paulo: Saraiva, 2007, p.211-230.

MENDONÇA, M. A.; FREITAS, F.; SOUZA, J. Tecnologia da informação e produtividade na indústria brasileira. Revista de Administração de Empresas, jan./mar. 2009, n. 1, v. 49. p.75-85, 2009.

MUROVEC, N.; PRODAN, I. Absorptive capacity, its determinants, and influence on innovation output: Cross-cultural validation of the structural model. Technovation, v. 29, n. 12, p. 859-872, dez. 2009.

NELSON, R. R. Por que as empresas diferem e qual é a importância disso? In: NELSON, R. R. (Ed.). As fontes do Crescimento Econômico. Campinas: Editora da Unicamp, 2006. p. 165-194.

NELSON, R.; SAMPAT, B. Making sense of institutions as a factor shaping economic performance, Journal of Economic Behavior and Organization, 44, p. 31-54, 2001

NELSON, R. R.; WINTER, S. G. Uma Teoria Evolucionária da Mudança Econômica. 1. ed. Campinas: Editora Unicamp, 2005.

OCDE/FINEP. Manual de Oslo: diretrizes para Coleta e Intepretação de dados sobre Inovação. Rio de Janeiro: Financiadora de Estudos e Projetos, p. 184.

PARANHOS, J.; HASENCLEVER, L. 2017. A Teoria da firma e empresa inovadora. Em: RAPINI, M.S.; SILVA, L.A.; ALBUQUERQUE, E. M. Economia da Ciência, Tecnologia e Inovação Fundamentos Teoricos e a Economia Global. Editora Prismas, 2017. SAPPRASERT, K.; 
CLAUSEN, T. H. Organizational innovation and its effects. Industrial and Corporate Change, v. 21, n. 5, p. 1283-1305, 2012.

SIMON, H. A. The Sciences of the Artificial. 3. ed. London: The MIT Press, 1996.

TEECE, D. J.; PISANO, G.; SHUEN, A. Dynamic capabilities and strategic management. Strategic Management Journal, v. 18, n. 7, p. 509-533, ago. 1997.

VIEIRA, K. P.; SANTOS, F.; OLIVEIRA, F. H. P. O pólo de tecnologia da informação de Belo Horizonte. Cadernos BDMG, n. 14, p. 1-130, abr. 2007..

VROMEN, J. J. Routines, genes and program-based behavior. Journal of Evolutionary Economics, v. 16, n. 5, p. 543-560, 2006.

ZAHRA, S. A.; GEORGE, G. Absorptive Capacity: A Review, Reconceptualization, and Extension. Academy of Management Review, v. 24, n. 2, p. 185-203, abr. 2002. 